\title{
What are Preoperative Predictors for Incidental Gallbladder Cancer after Routine Cholecystectomy?
}

Mitsugi Shimoda, Tsunehiko Maruyama, Kiyotaka Nishida, Kazuomi Suzuki, Tomoya Tago, Jiro Shimazaki, Shuji Suzuki

Department of Gastroenterological Surgery, Tokyo Medical University, Ibaraki Medical Center, Japan

\section{ABSTRACT}

Previous reports show that incidental gallbladder cancer (iGbC) occurs in less than $2 \%$ of patients after routine cholecystectomy. The aim of this retrospective study was to analyse the incidence and preoperative risk factors associated with iGbC at our department.

Patients and Methods: Between January 2006 and October 2016, a total of 480 cholecystectomies were performed, and we divided the cohort into two groups: iGbC Group (iGbC G) and non-Gb cancer group (nGbC G). Univariate and multivariate analyses assessing preoperative clinical and laboratory characteristics were performed in order to investigate the most significant risk factors for patients with iGb C.

Results: iGbC G was confirmed by histopathology in five patients, while 475 had no malignant disease. There were 3 females and 2 males in the iGBC group, with a median age of 62.7 years. According to the univariate analysis, adverse preoperative prognostic factors were lymphocyte percentage $(p=0.006)$ and neutrophil percentage $(p=0.016)$, while according to the multivariate analysis, lymphocyte percentage (odds ratio 0.76 ; $95 \% \mathrm{Cl} ; 0.59-0.96, p=0.023$ ) was a significant adverse prognostic factor.

Conclusion: Preoperative lymphocyte percentage is the most important risk factor foriGbC. Key words: laparoscopic, Incidental gallbladder cancer, cholecystectomy, lymphocyte

\section{INTRODUCTION}

Gallbladder cancer $(\mathrm{GbC})$ is a rare though notoriously lethal malignancy with marked ethnic and geographical variations. It is a common disease in countries such as Chile, Japan, India, Central Europe, Poland, Israel, and Southern Pakistan; however, it is uncommon in the United States (US), and more than $60 \%$ of the patients are females, usually aged over 65 years (1). Occasionally, Gallbladder (Gb) malignancy is found on pathological reports after routine cholecystectomy. Recent studies have reported an increase in incidental Gb cancer (iGbC), with approximately $50 \%-58 \%$ of all new GbC cases $(2,3)$ being now discovered incidentally. Currently, a laparoscopic cholecystectomy (LC) is standard procedure for the treatment of benign Gb diseases. In the US, more than a million LC are performed each year (4). For that reason, iGbC discovered during or following $\mathrm{LC}$ has been increasing in the last decade. Some authors have
Corresponding author: Mitsugi Shimoda, M.D., Ph.D. 3-20-1, Chuo, Ami, Ibaraki 300-0395, Japan

Tel: $+81-29-887-1161$

Fax: +81-29-840-2089

E-mail: mshimoda@tokyo-med.ac.jp 
reported an incidence of iGbC after LC of approximately $0.2 \%-2.1 \%(5,6)$.

In general, it is difficult to diagnose $\mathrm{GbC}$ in most routine cholecystectomies performed for a preoperative diagnosis of benign $\mathrm{Gb}$ diseases. There have been few reports on the predictive factors of iGbC. Those articles identified old age, female gender, Asian or African American heritage, elevated serum ALP and acute cholecystitis with jaundice as potential risk factors for iGbC (7-9).

In this retrospective study, we revealed the preoperative clinical and operative characteristics of iGbC compared with benign $\mathrm{Gb}$ diseases, and sought to find the risk factors.

\section{MATERIALSAND METHODS}

\section{Patient population and selection}

From January 2010 to October 2016, a total of 480 routine cholecystectomies were performed in our medical center, and the patients and their operational outcomes were retrospectively analyzed in this study.

Prior to surgery all patients followed a standard institutional protocol of clinical examination, blood tests, and urine screening of the abdomen. In all of patients, computed tomography, magnetic resonance imaging, and abdominal ultrasonography were routinely carried out as preoperative examinations. Finally, we propose a management strategy for acute cholecystitis according to the 2013 Tokyo Guidelines, which is available via http://www.jshbps.jp/en/guideline/tg13.html.

If there was a suspected $\mathrm{Gb} C$ in preoperative diagnosis, the patient was excluded from this study.

\section{Surgical procedure}

All operations were performed by a team of two or three surgeons who were adequately experienced in the open as well as the laparoscopic approach. Three or four ports were used in the LC procedure, one $10-\mathrm{mm}$ port (camera port) and three 5 -mm ports.

In conventional cholecystectomy, the $\mathrm{Gb}$ is dissected along the inner layer of the subserosal layer. On the other hand, the $\mathrm{Gb}$ is removed including the cystic plate by dissecting along the outer layer of the subserosal layer in whole layer cholecystectomy. These benign lesions include wall-thickening lesions such as adenomyomatosis and chronic cholecystitis, pedunculated polyps smaller than $15 \mathrm{~mm}$ or situated on the peritoneal side, as well as sessile polyps situated on the peritoneal side and smaller than $15 \mathrm{~mm}$ (10). When postoperative pathological examination reveals the presence of $\mathrm{Gb} C$ invading beyond the muscular layer, additional $\mathrm{Gb}$ bed resection and regional lymphadenectomy are considered.

\section{Study design}

We evaluated clinical and operative factors in all of the 480 patients. The patients were divided into two groups: the iGbC group (iGbCG), and non-Gb cancer group ( $\mathrm{nGbC}$ G). Clinical factors were selected and compared between the two groups. These included age, gender, body mass index (BMI), albumin (Alb), C-reactive protein (CRP), neutrophil-to-lymphocyte ratio (NLR), white cell count $(\mathrm{WBC})$, neutrophil ratio, lymphocyte ratio, Platelet count, Neutrophil / Lymphocyte ratio (NLR), C-reactive protein / albumin ratio (CAR), Platelet/ Lymphocyte ratio (PLR), operative factors (duration and amount of blood loss), length of hospital stay (LHS), LC (yes/no).

The study was approved by the research and ethics committee at the Tokyo Medical University, Ibaraki Medical Center (Number:16-35). The patients who completed follow-ups were also included in this study.

\section{Statistics}

Statistical analyses were performed with the SPSS statistical software package (version 13.0; SPSS Inc., Chicago, IL). Median was used to define laboratory parameters such as, age, gender, BMI, Alb, CRP, NLR, WBC, neutrophil ratio, lymphocyte ratio, Platelet count, NLR, CAR, PLR, operating time, amount of blood loss, and LHS. Univariate and multivariate analyses were performed in order to clarify the laboratory parameter and clinical factors most significantly associated with $\mathrm{iGbC}$ and $\mathrm{nGbC}$ G. Univariate analyses, Mann-Whitney U-test, and Fisher's exact test were utilized, and Odds ratios with $95 \% \mathrm{Cl}$ were calculated using logistic regression model analyses. $\mathrm{P}$ values of less than 0.05 were considered to be statistically significant.

\section{RESULTS}

From January 2006 to October 2016, 480 patients underwent routine cholecystectomies with a presumptive preoperative diagnosis of benign $\mathrm{Gb}$ disease. Five of the 480 cases were iGbC and 475 patients had benign $\mathrm{Gb}$ disease on histological findings.

\section{Characteristics of iGb C group}

There were 3 females and 2 males, and the median 
age was 62 years old (range: $50-88$ ). The preoperative diagnosis consisted of: 2 cases with Gb stones, 2 cases of cholecystitis with $\mathrm{Gb}$ stone, and one was $\mathrm{Gb}$ polyp. One patient had open cholecystectomy and 4 patients had LC. Two of the five cases had stage II disease, 2 were stage I and 1 case had stage III disease according to the pathological findings. The latter stage 3 case had subserosa invasion and lymph node metastasis (T2, N1, $\mathrm{MO})$, so we performed liver resection with additional lymph node dissection. One patient died from liver metastasis (table 1).

\section{Comparing iGbCG and $n G b C G$}

\section{Univariate analysis}

The percentage of lymphocytes was significantly lower in iGbCG than in $\mathrm{nGbC} G(\mathrm{p}=0.006)$, while the percentage of neutrophils was significantly higher in iGbCG than in nGbC G (odd ratio 0.88 ; $95 \% \mathrm{Cl}$; 0.79 $0.96, p=0.006$ : table 2). There were no significant differences between the two groups in terms of operative factors and length of hospital stay (table 3).

Table 1 - The characteristics of iGbC cases

\begin{tabular}{lccccccc}
\hline NO & Gender & Age & Pre.diag. & Acute Cholecystitis & depth & $\begin{array}{c}\text { Additional operation } \\
\text { (yes/no) }\end{array}$ & Death /Alive \\
\hline 1 & F & 78 & GS & - & $m$ & no & Alive \\
\hdashline 2 & F & 71 & GS & - & SS & no & Death \\
33 & F & 50 & CGS & - & SS & yes & Alive \\
\hdashline 4 & M & 67 & Polyp & - & m & no & Alive \\
\hline
\end{tabular}

GS: Gall bladder stone, Pre.diag., preoperative diagnosis, CGS: Cholecystitis with Gall bladder stone,

LC: laparoscopic cholecystectomy, OC: open cholecystectomy

Table 2 - The characteristics before surgery according to the procedure

\begin{tabular}{|c|c|c|c|}
\hline & iGbC G (n=4) & $n G b C$ G (n=421) & p \\
\hline Age (years) & $69.5(50-78)$ & $60.8(17-91)$ & $0.28^{*}$ \\
\hline Gender (F/M) & $3 / 1$ & $202 / 219$ & $0.31 \dagger$ \\
\hline $\mathrm{BMI}$ & $23.7(18.9-27.6)$ & $24.5(14.7-38.6)$ & $0.49^{*}$ \\
\hline $\mathrm{CRP}(\mathrm{mg} / \mathrm{dl})$ & $0.03(0.03-1.96)$ & $0.12(0.01-20.6)$ & $0.99^{*}$ \\
\hline Alb $(\mathrm{g} / \mathrm{dL})$ & $4.1(3.1-4.5)$ & $4.3(2.6-5.4)$ & $0.21^{*}$ \\
\hline WBC $\left(10^{3} / \mu \mathrm{L}\right)$ & $5.1(3.3-7.6)$ & $5.7(0.5-18.1)$ & $0.43^{*}$ \\
\hline Neutro. (Count) & $3523(2227-5320)$ & $3145(253-16290)$ & $0.94^{*}$ \\
\hline Lymph (Count) & $1061(405-1748)$ & $1857(163-5635)$ & $0.009^{*}$ \\
\hline PLt $\left(10^{4} / \mu \mathrm{L}\right)$ & $22.2(14.0-48.0)$ & $22.3(47.0-49.8)$ & $0.24^{*}$ \\
\hline NLR & $9.3(1.9-5.7)$ & $1.7(0.7-15.0)$ & $0.04^{*}$ \\
\hline CAR & $0.07(0.01-0.63)$ & $0.03(0.0-4.8)$ & $0.87^{\star}$ \\
\hline PLR & $0.26(0.19-0.35)$ & $0.12(0.03-1.69)$ & $0.11^{\star}$ \\
\hline
\end{tabular}

Showing medians and interquartile ranges.

*Tested by Mann-Whitney U-test. †Tested by Fisher's exact test. CRP:C-reactive protein, WBC: white cell count, Alb: Albumin, Neutro: neutrophil, Lymph: Iymphocyte, PLt: Platelet, NLR: Neutrophil / Lymphocyte ratio,CAR: C-reactive protein / albumin ratio, PLR: Platelet/Lymphocyte ratio

Table 3 - The outcomes according to the procedure

\begin{tabular}{lccc}
\hline & iGbC $\mathbf{G}(\mathbf{n}=\mathbf{4})$ & $\mathbf{n G b C} \mathbf{G}(\mathbf{n}=\mathbf{4 2 1})$ & $\mathbf{p}$ \\
\hline Operating time (min.) & $147(65-310)$ & $127(50-394)$ & $0.42^{\star}$ \\
\hdashline Blood loss (g) & $1(1-728)$ & $1(1-7084)$ & $0.62^{*}$ \\
\hline LHS (days) & $7.5(3-10)$ & $5(1-45)$ & $0.88^{*}$ \\
\hline
\end{tabular}

Showing medians and interquartile ranges.

*Tested by Mann-Whitney U-test. †Tested by Fisher's exact test.

LC: laparoscopic cholecystectomy, LHS: length of hospital stay 


\section{Multivariate analysis}

Lymphocyte ratio was the only significant adverse prognostic factor in multivariate analysis (odd ratio 0.76; $95 \% \mathrm{Cl}$; 0.59-0.96, $\mathrm{p}=0.023$ : table 4).

\section{DISCUSSION}

Cholecystectomy is one of the most common surgical procedures performed around the world. Technical advances in preoperative imaging systems have contributed to earlier detection of GbC. However, previous reports described incidental gallbladder cancer (iGbC) still occurs in $0.5 \% \sim 1.6 \%$ on histological findings after cholecystectomy. In our study, 5 cases $(1.0 \%)$ of iGbC were confirmed. Even though several GbC risk factors have been described, many of them are based on epidemiology or small size of cohorts in advanced tumours.

$\mathrm{Gb} C$ is confirmed by the pathological findings, proceeding with iterative surgery for $\mathrm{RO}$ resection is necessary. $\mathrm{RO}$ resection is the most important positive factor for overall survival of iGb C. It was affected for overall survival patients with iGb $C$. The iterative surgery varies depending on the depth of invasion ( $T$ stage) of the cancer. For a T1a tumour, simple cholecystectomy is the standard procedure, whereas for a T1b tumour, cholecystectomy with lymph node dissection is the standard procedure. For T2-3 and more advanced tumours, liver resection including the gallbladder bed or bisegmentectomy ( $\mathrm{V}$ and $\mathrm{IVb}$ ) and lymph node dissection are recommended. Extrahepatic bile duct resection is not performed uniformly, and is somewhat controversial in the surgical treatment of Gb C (11). Patient's refusal of radical surgery after initial cholecystectomy detecting $\mathrm{iGbC}$ is one of the more difficult problems encountered in clinical practice (11). Even further, we suggest that preoperative cancer detection is a more important factor for rescuing patients with iGb C.

Unfortunately, few reports focused on predictors of iGbC, which were elder patients, female gender, dilated bile duct, and acute cholecystitis with jaundice $(8,9,12)$. However, female and advanced age are considered common risk factors for cancer and could not be proved as particular predictors for iGbC. Furthermore, when if patients have bile duct dilatation or jaundice, surgeons should carefully diagnose and pay attention to detecting cancers using advanced imaging modalities. In addition, we propose that such patients should never be selected for routine cholecystectomy. We first revealed that the preoperative peripheral lymphocyte and neutrophil percentage were shown to be carcinogenic risk factors in iGbC. Moreover, preoperative low lymphocyte percentages had the strongest association with iGbC, and we suggest that it might be a different type of predictor than previous reports.

Lymphocytes play an important role in antitumour immune responses and a persistent immunosuppressive state is associated with a high probability of recurrence (13). A single malignant cell may have multiple tumour-specific antigens. For example, cluster of differentiation-4 (CD4)+and CD8+ T-cells initiate anti-tumour response and are also tumourspecific antigens. The activation of those cells requires the presentation of antigenic peptides on professional antigen-presenting cells. Actually, a low CD4+ and CD8+ T-cells infiltration detected in the tumour correlates with a poor prognosis for $\mathrm{GbC}$ (14). Such an immunological response could be reflecting a preoperative cancer status in patients with GbC. However, routine measurement of CD4+ and CD8+ T-cells is not costefficient. On the other hand, measurement of preoperative lymphocyte percentage could be obtained from routine blood laboratory testing, and is more cost-effective than measuring CD4+and CD8+ T-cells. It is suggested that it can be easily used as a predictor of iGbCin the future.

Nowadays, several reports describe that inflammatory factors such as NLR and GPS are related to poor prognosis factor in patients with several cancers (15-17). Perioperative changes in immune response are among the predictors of therapeutic outcome after curative resection for hepatobiliary cancer $(18,19)$.

Though high neutrophil percentage and low lymphocyte percentage were selected as potential predictors for iGbC in this study, NLR did not show any significance as a cancer predictor. Other inflammatory markers such as, CAR and PLR also did not qualify as a possible predictor for iGbC.

Table 4 - Multivariate Analysis clinical and operative factors according to the procedure

\begin{tabular}{lccccc}
\hline & iGbCG $(\mathbf{n}=\mathbf{4})$ & nGbCG $(\mathbf{n}=421)$ & Odd ratio & $\mathbf{9 5 \%}$ C.I. & $\mathbf{p}$ \\
\hline Lymph (count) & $1061(405-1748)$ & $1857(163-5635)$ & 1.002 & $1.000-1.005$ & $0.025 \#$ \\
\hline
\end{tabular}

\#Tested by logistic regression model analyses 


\section{CONCLUSION}

The preoperative peripheral blood percentage of lymphocytes positively correlates with iGbC after routine cholecystectomy. Measurement of preoperative peripheral blood lymphocytes may be useful for cancer screening of patients planning for cholecystectomy.

\section{Conflicts of Interest Statement}

The authors declare no conflicts of interest in this study.

\section{REFERENCES}

1. Tewari M, Kumar S, Shukla S, Shukla HS. Analysis of wedge resection of gallbladder bed and lymphadenectomy on adequate oncologic clearance for gallbladder cancer. Indian J Cancer 2016; 53(4):552-57. doi: 10.4103/ijc.IJC_88_17

2. Agarwal AK, Kalayarasan R, Javed A, Gupta N, Nag HH. The role of staging laparoscopy in primary gall bladder cancer--an analysis of 409 patients: a prospective study to evaluate the role of staging laparoscopy in the management of gallbladder cancer. Ann Surg 2013;258(2):318-23. doi: 10.1097/SLA.0b013e318271497e

3. Shih SP, Schulick RD, Cameron JL, Lillemoe KD, Pitt HA, Chot MA, et al. Gallbladder cancer: the role of laparoscopy and radical resection. Ann Surg. 2007;245(6):893-901.

4. Tian YH, Ji X, Liu B, Yang GY, Meng XF, Xia HT, et al. Surgical treatment of incidental gallbladder cancer discovered during or following laparoscopic cholecystectomy. World J Surg. 2015;39(3): 746-52. doi: 10.1007/s00268-014-2864-9.

5. Yamamoto H, Hayakawa N, Kitagawa Y, Katohno Y, Sasaya T, Takara $D$, et al. Unsuspected gallbladder carcinoma after laparoscopic cholecystectomy. J Hepatobiliary Pancreat Surg. 2005;12(5):391-8.

6. Kwon AH, Imamura A, Kitade H, Kamiyama Y. Unsuspected gallbladder cancer diagnosed during or after laparoscopic cholecystectomy. J Surg Oncol 2008;97(3):241-5. doi: 10.1002/jso.20944

7. Pitt SC, Jin LX, Hall BL, Strasberg SM, Pitt HA. Incidental gallbladder cancer at cholecystectomy: when should the surgeon be suspicious? Ann Surg 2014;260(1):128-33.

8. Muszynska C, Lundgren L, Lindell G, Andersson R, Nilsson J, Sandström $P$, et al. Predictors of incidental gallbladder cancer in patients undergoing cholecystectomy for benign gallbladder disease: Results from a population-based gallstone surgery registry.
Surgery. 2017;162(2):256-263. doi: 10.1016/j.surg.2017.02.009. Epub 2017 Apr 8 .

9. Ahn Y, Park CS, Hwang S, Jang HJ, Choi KM, Lee SG. Incidental gallbladder cancer after routine cholecystectomy: when should we suspect it preoperatively and what are predictors of patient survival? Ann Surg Treat Res 2016;90(3):131-8. doi: 10.4174/ astr.2016.90.3.131

10. Ome $\mathrm{Y}$, Hashida $\mathrm{K}$, Yokota M, Nagahisa $\mathrm{Y}$, Okabe M, Kawamoto K. Laparoscopic approach to suspected T1 and T2 gallbladder carcinoma. World J Gastroenterol 2017;23(14):2556-65. doi: 10.3748/ wjg.v23.114.2556

11. Choi KS, Choi SB, Park P, Kim WB, Choi SY. Clinical characteristics of incidental or unsuspected gallbladder cancers diagnosed during or after cholecystectomy: a systematic review and meta-analysis. World J Gastroenterol 2015;21(4):1315-23.

12. Dorobisz T, Dorobisz K, Chabowski M, Pawłowski W, Janczak D, Patrzałek $\mathrm{D}$, et al. Incidental gallbladder cancer after cholecystectomy: 1990 to 2014. Onco Targets Ther 2016;9:4913-6.

13. Bishara S, Griffin M, Cargill A, Bali A, Gore ME, Kaye SB, et al. Pre-treatment white blood cell subtypes as prognostic indicators in ovarian cancer. Eur J Obstet Gynecol Reprod Biol 2008;138(1):71-5. doi: 10.1016/..ejogrb.2007.05.012

14. Nakakubo $\mathrm{Y}$, Miyamoto M, Cho Y, Hida Y, Oshikiri T, Suzuoki M, et al. Clinical significance of immune cell infiltration within gallbladder cancer. Br J Cancer 2003;89(9):1736-42. doi: 10.1038/sj.bjc.6601331

15. Hu D, Zhang H, Lin X, Chen G, Li C, Liang B, et al. Elevated preoperative neutrophil-to-lymphocyte ratio can predict poor survival in early stage gastric cancer patients receiving radical gastrectomy: The Fujian prospective investigation of cancer (FIESTA) study. J Cancer 2017;8(7):1214-22. doi: 10.7150/jca.18707 eCollection 2017.

16. Sugiura T, Uesaka K, Kanemoto H, Mizuno T, Okamura Y. Elevated preoperative neutrophil-to-lymphocyte ratio as a predictor of survival after gastroenterostomy in patients with advanced pancreatic adenocarcinoma. Ann Surg Oncol 2013;20(13):4330-7. doi: 10.1245/s10434-013-3227-8 Epub 2013 Aug 28.

17. Ishizuka M, Kubota K, Kita J, Shimoda M, Kato M, Sawada T. Impact of an inflammation-based prognostic system on patients undergoing surgery for hepatocellular carcinoma: a retrospective study of 398 Japanese patients. Am J Surg 2012;203(1):101-6. doi: 10.1016/j.amjsurg.2010.09.030

18. Fujiwara $Y$, Shiba H, Furukawa K, lida T, Sakamoto T, Gocho T, et al. Perioperative change in white blood cell count predicts outcome of hepatic resection for hepatocellular carcinoma. J Hepatobiliary Pancreat Sci 2010;17(6):892-7. doi: 10.1007/s00534-010-0290-4

19. Haruki K, Shiba H, Fujiwara Y, Furukawa K, Wakiyama S, Ogawa M, et al. Perioperative change in peripheral blood monocyte count may predict prognosis in patients with colorectal liver metastasis after hepatic resection. J Surg Oncol 2012;106(1):31-5. 